\title{
Time and Space Integrating Acousto-Optic Folded Spectrum Processing for SETI
}

\author{
K. Wagner ${ }^{1}$ and D. Psaltıs \\ California Instıtute of Technology
}

\begin{abstract}
Time and space integrating folded spectrum techniques utilizing acousto-optic devices (AOD) as 1-D input transducers are investigated for a potential application as wideband, high resolution, large processing gain spectrum analyzers in the search for extra-terrestrial intelligence (SETI) program. The space integrating Fourier transform performed by a lens channels the coarse spectral components diffracted from an AOD onto an array of time integrating narrowband fine resolution spectrum analyzers The pulsing action of a laser diode samples the interferometrically detected output, aliasing the fine resolution components to baseband, as required for the subsequent $C C D$ processing. The raster scan mechanism incorporated into the readout of the CCD detector array is used to unfold the 2-D transform, reproducing the desired high resolution Fourier transform of the input signal.
\end{abstract}

\section{Introduction}

The Search for Extra-Terrestrial Intelligence (SETI) is an ambitious program to examine 773 of the nearest solar type stars as well as the entire celestial sphere for any evidence of intelligent microwave activity, either beamed at us or leaked from a civilization's internal communications (Ref 1$)$. The interstellar distances are so large and the resulting $1 / R^{2}$ attenuation is so great that the receiver sensitivity to a signal buried in cosmic background noise must be as large as possible. Without a priori knowledge as to the transmitted signal structure, ideal matched filters cannot be constructed; however, a good assumption is that a transmitted beacon would

\footnotetext{
${ }^{1}$ Graduate student in Electrical Engineering.
}

have a narrow band highly coherent structure in a sea of broadband white noise. Thus a narrow bandpass filter, with resolution as small as $1-100 \mathrm{~Hz}$, could achieve enough processing gain to detect the presence of an intelligent $\mathrm{CW}$ signal. Since the preferred frequency for interstellar microwave communication could conceivably lie anywhere from 0.1 to 10 or $100 \mathrm{GHz}$, then from $10^{8}$ to $10^{11}$ frequency measurements must be made for each resolvable spot on the celestial sphere observed by the radio telescope. If these measurements were performed sequentially with a swept local oscillator tuned bandpass filter, then as much as a million years of observation time could be required $A$ much more practical approach would be to build a wideband, high resolution spectrum analyzer with a million or more parallel channels of band pass filters, thereby cutting down observation time to on the prder of a year. Currently two approaches are being pursued, 
a digital multi-channel spectrum analyzer (MCSA) (Ref. 1) with a tree configuration of DFT and FFT subunits, and an analog acousto-optic folded spectrum processor as reported in this article

The advanced state of the art of wideband acousto-optic (AO) Bragg cells make them ideal candidates for entering 1-D data into an optical signal processing system, and the Fourier transforming property of a lens makes the implementation of real-time spectrum analyzers with 1000 resolvable frequencies routine. In this article we explore some possible approaches to performing narrow band time integrating (TI) spectrum analysis on each of the $10^{3}$ channels of a space integrating (SI) AO spectrum analyzer, thereby obtaining a high resolution folded spectrum through time and space integration (TSI).

\section{Folded Spectrum Processing}

The concept of the Folded Spectrum was introduced by Thomas (Ref. 2), who showed that the 2-D Fourier transform of a raster scanned signal was an orthogonally raster scanned version of the long 1-D spectrum of the original signal. This is schematically illustrated in $F_{1} g .1$. The folded spectrum is ideal for an optical signal processing system since it utilizes the two available spatial coordinates to perform a high resolution spectrum analysis that would be impossible in a 1-D system due to the limitations in space bandwidth product of 1-D devices. This processing operation can be performed in real time by raster scanning a very long input signal $s(t)$ onto a 2-D spatial light modulator (SLM), and using the 2-D space integrating Fourier transform operation performed by a sphencal lens. After the complete 2-D raster is formed, coherent light is passed through the SLM and the resulting amplitude distribution $f(x, n)$ is Fourier transformed by the lens. The power spectrum $\left|F\left(u_{c}, v_{f}\right)\right|^{2}$ is detected by a 2-D charge coupled device (CCD) detector array at the back focal plane. The orthogonally onented raster readout of the CCD turns the 2-D power spectrum back into a high resolution 1-D power spectrum $|S(f)|^{2}$, at a rate of up to 60 such transformations each second. However, the avallability of highly mature CCD devices with up to $2000 \times 2000$ pixels can not be fully utilized because of the lower resolution and dynamic range of currently available 2-D SLMs. Thus an architecture for performing the high resolution folded spectrum operation without the need for 2-D SLMs is highly desirable.

Turpin (Ref. 3) and Kellman (Ref. 4) have suggested such an approach utillzing acousto-optic devices (AODs) in an interferometric time integratıng configuration. In this approach a 2-D array of local oscillators with coarse and fine frequen- cies in $x$ and $y$ is produced by orthogonal AODs to which fast and slow chirps are applied respectively. When a frequency component of the input signal matches that of a particular local oscillator, a baseband heterodyne component is generated, and a time integrated peak is created at that location, proportional in amplitude to the amplitude of the corresponding Fourier component. Since this approach involves interferometric detection, a bias term will be generated due to the local oscillator reference and a signal dependent bias will occur uniformly throughout the detector array. These components can be separated by placing the desired heterodyne term on a spatial carrier. However in a white noise environment, as envisioned for the SETI search with a weak narrow band CW peak, the signal dependent bias term will produce a large bias because all its frequency components are incoherently detected at each CCD pixel, thereby using up the available $C C D$ dynamic range. Thus the 2-D time integrating technique for folded spectrum processing suffers in a multi-signal environment from a large signal dependent bias term, and places too large a requirement on CCD dynamic range.

A hybrid approach that utlizes the 1-D space integrating Fourier transformation property of a lens in one dimension followed by an interferometric time integrating fine resolution spectral analysis can combine the best features of the 2-D SI and 2-D TI approaches to folded spectrum processing. This approach utilizes a standard space integrating acoustooptic spectrum analyzer (Fig. 3) to perform the coarse spec. tral analysis without introducing a signal dependent bras term. The number of resolvable coarse frequency bins is limited by the time bandwidth product of the AOD and the input apodization to approximately $T_{a} B=1000$ The doppler frequency shift associated with acousto-optıc Bragg diffrac. tion produces a linear spatial dependence of the temporal frequency at the output spectrum that must be converted to baseband at all positions for further time integrating processing on a CCD. Bader (Refs. 5,6) proposed a way to do this with a spatially distributed local oscillator (DLO) reference wave which is produced with an auxiliary AOD that has a repettive wideband signal applied to its transducer. Interferometric detection of the signal and reference Fourier spectra produces narrow band baseband signal components at each output location. These narrow band signals can be used to produce a time integrating fine frequency analysis in the orthogonal direction on a 2-D CCD through the use of the chirp transform algorithm by premultiplying each narrow band signal with a slow chirp and then convolving with a matched chirp using another AOD. This type of system has a less severe signal dependent bias build up problem because only signals within a single coarse frequency bin contribute any bias to that bin. In this article we will present an alternative approach to tıme and space integrating folded spectrum processing. 
This method, which utilizes pulsed laser diodes, was originally proposed by Psaltis (Refs. 7, 8) and is lllustrated schematically in Fig. 2. A very long wideband signal is to be optically processed utilizing an acousto-optic device as the input transducer. However, since the limited time-bandwidth product of the AOD will not allow the entıre signal to be entered into the AOD simultaneously, we will successively slide overlapping portions of the long signal into the AOD and freeze the acoustic motion by pulsing the illuminating laser diode with a very narrow pulse. For each laser diode pulse we have a 1-D spatial representation of part of the input signal as an optically coherent wavefront, and on successive pulses we will transduce successive portions of the input signal into the optical system. Thus we have transformed the long signal into a 2-D raster of space $(x)$, and time or pulse number $(n)$. The transformation performed by the AOD lluminated with a repetitively pulsed laser diode is given by

$$
f(x, n)=\sum_{n=0}^{N-1} s(t-x / \nu) \operatorname{rect}(x / A) \delta(t-n T)
$$

In this expression $v$ is the acoustic velocity of the traveling wave AOD, $A$ is the AOD width, and $T$ is the laser diode pulse repetition interval. If $A>\nu T$, the raster is oversampled, which means that successive raster lines are overlapping. We perform the desired long 1-D Fourier transform on the input signal $s(t)$, with a 2-D transform on its oversampled 2-D raster representation $f(x, n)$. Because of the separability of the multidimensional Fourier kernel, a 2-D transform can be performed as $N$ 1-D transforms along one axis, followed by $N$ 1-D transforms along the orthogonal axis This cascaded system can be implemented by performing a spatial Fourier transform with a lens for each of the $N$ laser diode pulses, producing an intermediate data set $F_{x}(u, n)$, which is also a space-tıme raster. This is followed by an array of time integratıng Fourier transform modules that utlize the second spatial dimension. $(y)$ to perform the desired accumulation of partial products. After a succession of $N$ laser diode pulses the full folded spectrum is produced in a 2-D format on a CCD detector array. The 2-D spectrum can be very simply unfolded back into the desired long 1-D spectrum by utilizing the raster readout mechanism incorporated in the CCD itself. The resulting system utilizes the mature component technologies of acousto-optics, charge coupled devices and laser diodes to produce a valuable real-tıme signal processing module for high resolution spectral analysis.

\section{Space Integrating Spectrum Analyzer With a Pulsed Laser Diode}

The operation of a space integrating (SI) acousto-optic spectrum analyzer is explained with reference to Fig. 3 A collimated coherent optical plane wave of wavelength $\lambda$ is incident upon the AOD at the Bragg angle,

$$
\Theta_{B}=-\sin ^{-1} \lambda / 2 \Lambda_{0} \sim-\lambda f_{0} / 2 \nu
$$

in order to maximize the optical power coupled into the first order diffracted beam. In this expression $f_{0}$ is the transducer center frequency, $\Lambda_{0}=v / f_{0}$ is the midband acoustic wavelength, and $v$ is the acoustic velocity. A band-limited signal is mixed to the AOD center frequency $f_{0}$, producing an octave bandwidth signal $s(t)$, that is amplified and applied to the piezoelectric transducer, thereby launching an acoustic wave replica of $s(t)$ into the photo-elastic medium. The acoustic wave creates a traveling wave volume index pertubation through the photo-elastic effect, which Bragg diffracts some of the incident light into the first order diffracted optical replica of a single sideband version of the portion of the signal $s(t)$ that is currently within the AOD aperture $A$. In the frequency domain this can be viewed as each Fourier component $S(f)$ of the signal $s(t)$ within the AOD bandwidth $B=2 f_{0} / 3$, producing a diffracted plane wave of aperture $A$ propogating at an angle $\theta_{d} \sim \lambda f / 2 v$ with respect to the optic axis, doppler shifted by the frequency $f$ due to the acoustic wave motion. This diffracted field propogates through a distance $F$ and is incident on a Fourier Transforming lens of focal length $F$, so that at a distance $F$ behind the lens the spatial Fourier transform of the signal within the AOD is formed. At the back focal plane the field observed is

$$
\begin{aligned}
F\left(x^{\prime}, t\right)= & \int_{A} s(t-x / v) \mathrm{e}^{-\jmath 2 \pi x x^{\prime} / \lambda F} d x \mathrm{e}^{j \omega t} \\
= & \int_{A}\left[\int_{B} S(f) \mathrm{e}^{2 \pi f(t-x / v)} d f\right] \\
& \times \mathrm{e}^{-\jmath 2 \pi x x^{\prime} / \lambda F} d x \mathrm{e}^{j \omega t} \\
= & \int S(f) \mathrm{e}^{2 \pi f t} \\
& \times A \operatorname{sinc}\left[A\left(x^{\prime} / \lambda F-f / v\right)\right] d f \mathrm{e}^{j \omega t} \\
= & {\left[v S\left(x^{\prime} \nu / \lambda F\right) \mathrm{e}^{j 2 \pi v x^{\prime} t / \lambda F} \mathrm{e}^{j \omega t}\right] } \\
& * A \operatorname{sinc}\left(A x^{\prime} / \lambda F\right)
\end{aligned}
$$

In this expression $x$ is the spatial coordinate along the axis of acoustic propogation, $x^{\prime}$ is the spatial coordinate at the Fourier plane, and $\omega=2 \pi c / \lambda$ is the optical radian frequency. The symbol * represents the convolution operation. We have 
neglected effects of input apodization, acoustic attenuation, anisotropic diffraction and band limitation, as well as other higher order effects. The exact doppler temporal phase history of each Fourier component is reproduced at the back focal plane, but the spatial representation of the spectrum is blurred by the Fourier transform of the finite aperture function of width $A$ thereby limiting the effective frequency resolution to $\delta f=1 / T_{a}=v / A$, where $T_{a}$ is the acoustic transit time across the aperture. This shows that the number of independent analog samples stored within the AOD at any particular instant, which is given by the time bandwidth product $T_{a} B$, also gives the effective number of independent frequencies that can be determined in the spatial Fourier transform plane. The actual frequency resolution is limited by the AOD finite aperture sinc convolved with the transforms of the optical and acoustic apodization functions, in order to produce an overall blur spot $Q\left(x^{\prime} / \lambda F\right)$ which yields somewhat worse frequency resolution than $1 / T_{a}$. Normal operation of an acousto-optic spectrum analyzer involves the square law detection process on a 1-D array of integrating photosensors, which incoherently accumulate charge for a time $T^{\prime}$ in order to improve the signal to noise ratio.

$$
\begin{aligned}
F\left(x^{\prime}\right) & =\int_{0}^{T^{\prime}}\left|F\left(x^{\prime}, t\right)\right|^{2} d t \\
& =\int_{0}^{T^{\prime}}\left|S\left(x^{\prime} \nu / \lambda F\right) * Q\left(x^{\prime} / \lambda F\right)\right|^{2} d t
\end{aligned}
$$

The output of this type of spectrum analyzer is a power spectrum of the signal $s(t)$ with a resolution limited by the blur spot $Q(u)$, with a normalized spatial frequency variable $u=x^{\prime} / \lambda F$. For a single sinusoid of frequency $f^{\prime}$ input to the spectrum analyzer, $s(t)=2 a \cos \left(2 \pi f^{\prime} t\right)$, with a single sided temporal Fourier spectrum given by $S(f)=a \delta\left(f-f^{\prime}\right)$, the time integrated output of the spectrum analyzer is given by

$$
F(u)=T^{\prime}|a|^{2} Q^{2}\left(u-f^{\prime} / v\right)
$$

A heterodyne detection technique can be employed by the addition of a simple plane wave reference beam. The instantaneous output of such an interferometric spectrum analyzer has a linear spatial dependence of the doppler induced temporal frequency that is given by

$$
\begin{aligned}
F(u, t)= & \left|\left[S(u v) \mathrm{e}^{\prime 2 \pi v u t} \mathrm{e}^{j \omega t}\right] * Q(u)+r \mathrm{e}^{j \omega t}\right|^{2} \\
= & \left|S(u v)^{*} Q(u)\right|^{2}+|r|^{2} \\
& +2 r[|S(u v)| \cos (2 \pi v u t+\Omega(u v))] * Q(u)
\end{aligned}
$$

where the amplitude and phase of the complex spectrum $S(f)=$ $|S(f)| \mathrm{e}^{\prime \Omega(f)}$ are reproduced as temporal modulations of each resolved frequency bin which is spatially blurred due to the apodization. For the case of the single sinusoldal input tone at a frequency $f^{\prime}$, with amplitude $|a|$ and phase $\Omega$, the heterodyne detected instantaneous output is

$$
\begin{aligned}
F(u, t)= & \left|a Q(u-f / v) \mathrm{e}^{j 2 \pi f^{\prime} t} \times \mathrm{e}^{j \omega t}+r \mathrm{e}^{j \omega t}\right|^{2} \\
= & |a|^{2} Q^{2}\left(u-f^{\prime} / v\right)+|r|^{2} \\
& +2|a| r Q\left(u-f^{\prime} / v\right) \cos \left(2 \pi f^{\prime} t+\Omega\right)
\end{aligned}
$$

The temporal modulation on the last term thus reproduces the input sinusold in both amplitude and phase, and could be used as the input to a fine resolution time integrating processor if we first heterodyne it to baseband. A repetitively pulsed light source is a simple and effective means of accomplishing this as long as the pulse width $\tau$ is less than the inverse of the bandwidth of $s(t)$, and much greater than the optical period of oscillation. When these conditions are satisfied we can consider the laser diode pulsed modulation to be approximated by a series of delta functions separated by a time $T$.

$$
\begin{aligned}
p(t) & =\sum_{n} \operatorname{rect}\left[\frac{t-n T}{\tau}\right] \\
& =\sum_{n} \delta(t-n T)
\end{aligned}
$$

This series of delta functions will act as sampling impulses on the interferometrically detected temporal modulation present at the output plane. It is convenient to consider the input temporal frequency to be broken up into a coarse and fine part by choosing the closest harmonic of the laser diode pulse repetition frequency as the coarse term and the offset from this frequency as the band-limited fine resolution component. Thus we substitute $f^{\prime}=k / T+\delta$ into Eq. (6), where $k=\bmod _{1 / T} f^{\prime}$ is an integer and $\delta=\operatorname{res}_{1 / T} f^{\prime}<1 / T$. For the case of repetitively pulsed laser illumination the heterodyne output of the AO spectrum analyzer for a single applied sinusordal tone, is given by

$$
\begin{aligned}
F(u, t)= & {\left[|a|^{2} Q^{2}(u-(k / T+\delta) / v)\right.} \\
& +|r|^{2}+2|a| r Q(u-(k / T+\delta) / v) \\
& \times \cos (2 \pi(k / T+\delta) t+\Omega)] p(t)
\end{aligned}
$$


The fine frequency component $\delta$ will not move the blur spot $Q(u)$ by a significant amount and can be dropped from within $Q(u)$. The coarse frequency component $k / T$ will be sampled by the laser diode pulses at times $t=n T$ resulting in an argument withın the cosine function of $2 \pi n k$, with $n$ and $k$ both integers, so that this term can be dropped leaving only the fine frequency modulation. Thus for the $n$th laser diode pulse at time $t=n T$ the sampled output can be approximated as

$$
\begin{aligned}
F(u, n T)= & |a|^{2} Q^{2}(u-k / T v)+|r|^{2} \\
& +2|a| r Q(u-k / T v) \cos (2 \pi \delta n T+\Omega)
\end{aligned}
$$

Thus for a single sinusoidal input the interferometric output of the space integrating spectrum analyzer 1lluminated with a series of delta functions can be seen to consist of a signal dependent bias term located at the position corresponding to the coarse frequency component, a uniform reference bias term and a sampled interferometrically detected spot located at the coarse frequency locus and oscillating in time at the fine frequency $\delta$, with phase $\Omega$. This effect of the sampling pulse train is usually referred to as aliasing and is considered undesirable. It indicates that the sampling rate is too low for the given signal bandwidth, and it results in different parts of the signal spectrum folding over on top of each other thereby producing invalid signal components. However in this case, the coarse frequency channeling of the SI spectrum analyzer effectively separates in space the various frequency components that could alias with each other. It is important to make sure that the sampling rate is fast enough to avoid aliasing of the bandlimited signals within each coarse frequency blur spot $Q(u)$. Since the bandwidth of the signal information within each blur spot is increased over $1 / T_{a}$ due to the apodization effects, we will assume that an appropriate bandwidth would be $2 / T_{a}$. To adequately sample these band-limited signal components the Nyquist criterion indicates that we should sample at least twice as fast as the highest frequency signal component present; thus the minimum sampling rate should be $1 / T>4 / T_{a}$ This indicates that the laser diode should be pulsed at least 4 times as an acoustic signal transits across the laser beam aperture illuminating the AOD in order to produce the appropriately oversampled space-time raster

\section{Time Integrating DFT Based Fine Resolution Processor}

A narrow band fine resolution spectrum analysis operation requires the coherent accumulation of data samples for a period at least as long as the inverse spectral resolution. For this reason an SI AO spectrum analyzer is limited in resolution to the inverse aperture time of the AOD In order to obtain a higher resolution spectrum analysis with AODs a time integrating (TI) approach must be utilized. Coherent accumulations can be carried out on an integrating charge coupled device (CCD) detector array as long as the thermally generated detector dark current and optical bias contributions do not saturate the desired signal components. With typical CCD device defect densities, thermal generation rates and signal well capacities, it should be possible to integrate for a full second, obtaining $1 \mathrm{~Hz}$ resolution capabilities, and with cooled detectors it would be possible to integrate even longer For the SETI program these resolution capabilities should be more than sufficient since spectral coherence of better than $1 \mathrm{~Hz}$ would be very difficult to obtain after propogating through the interstellar medium and being doppler shifted by both transmitter and receiver motions.

The required operation that needs to be performed on the output of each coarse frequency bin of the interferometric SI spectrum analyzer with a pulsed LD reported in the previous section is a narrow band discrete Fourier transform (DFT). The DFT is the discrete version of the Fourier transform as is appropriate for sampled data streams such as that produced by the interferometric spectrum analyzer illuminated by a pulsed laser diode. If we examine the third term in Eq. (9) we can see that for each input sinusoid we have a sampled fine frequency temporal oscillation $s(n)$ that we want to perform a DFT on. The DFT is defined for such a sampled input signal $s(n)$ as

$$
S(m)=\sum_{n}^{N} s(n) \mathrm{e}^{-/ 2 \pi n m / N}
$$

This operation is a vector matrix multiplication, where the sampled input signal $s(n)$ is the input vector, the DFT kernel is the matrix, and $S(m)$ is the output vector. A possible time integrating interferometric optical implementation using a pulsed laser diode is shown schematically in Fig 4. The light from a repetitively pulsed laser diode is split into two by a beam splitter. One arm illuminates an acousto-optic modulator that has a narrowband signal $s(t)$ applied to its transducer sampled by the narrow pulse of the laser diode producing a temporal modulation $s(n T)$. In the other arm, a reference AOD with aperture $T$, equal to the LD pulse repetition interval, is schlieren imaged onto the output $C C D$ array where it is interferometrically combined with the spatially uniform signal beam producing a sinusoidal spatial fringe pattern. With each laser diode pulse a new column of the DFT matrix is appled to the reference AOD transducer, and propogates across the aperture to align with the CCD at which time its motion is frozen by the LD pulse, thereby imaging that column onto the CCD. Thus the reference signal is analogous to a raster scanned version of the DFT matrix, except that along the columns a continuous representation is used which is later 
sampled by the spatially discrete CCD pixel structure. Since the successive columns of the DFT matrix represent sinusoids of successively higher frequencies the reference waveform is a stepped frequency chirp, with the constraint that each new frequency phase resets to zero phase each $T$ The reference signal starts at frequency $f$ and frequency steps by $\Delta f$ each time $T$ which is analytically represented as

$$
r(t)=\sum_{n=0}^{N-1} \operatorname{rect}\left[\frac{t-n T}{T}\right] \times \cos [2 \pi(f+n \Delta f)(t-N T)]
$$

This signal propogates in the $y$ direction at a velocity $v$, and aligns with the AOD and CCD apertures at the times of the laser diode pulses as given in Eq. (7). The spatial modulation diffracted by the AOD on the $n$th pulse is given by

$$
\begin{aligned}
r(y, n) & =r(t-y / \nu) \operatorname{rect}[y / \nu T] \delta(t-n T) \\
& =\operatorname{rect}[y / V T] \mathrm{e}^{2 \pi(f+n \Delta f) y / \nu}
\end{aligned}
$$

which is seen to be a finite aperture plane wave with an angular spatial frequency that is stepped linearly with the pulse number $n$. The interferometrically detected intensity pattern on the $n$th LD pulse is found by combining weighted signal and reference beams. The signal beam propogates at an angle $\theta$ with respect to the starting frequency of the reference, resulting in a spatial carner of spatial frequency $\alpha=\sin \theta / \lambda$.

$$
\begin{aligned}
I(y, n)= & \left|G s(n T) \mathrm{e}^{j 2 \pi(\alpha+f / v) y}+b r(y, n)\right|^{2} \\
= & |G|^{2}+|b|^{2}+2 G b s(n T) \\
& \times \cos [2 \pi(n \Delta f y / v+\alpha y)]
\end{aligned}
$$

By time integrating over $N$ successive LD pulses we obtain a discrete summation of the interferometrically detected charge distributions due to each pulse.

$$
\begin{aligned}
I(y)= & N\left(|G|^{2}+|b|^{2}\right) \\
& +2 G b \sum_{n=0}^{N-1} s(n T) \cos [2 \pi(n \Delta f y / v+\alpha y)]
\end{aligned}
$$

This can be recognized as the discrete cosine transform of the sequence $s(n T)$, with an analysis bandwidth of $\Delta f$, on a spatial carrier of frequency $\gamma=\alpha+(N-1) \Delta f / 2 v$. The carrier allows a bandpass filter operation to remove the bias terms, and the full complex DFT can be reconstructed by measuring the phase angle of the carrier for each resolvable frequency component. The CCD is composed of an array of pixels that must have sufficient resolution to adequately sample the spatial fringe structure under the DFT transform. For a pixel size $\Delta y$, the number of pixels per cycle of the fringe structure is $1 / \gamma \Delta y$, and should be adjusted to 4 to 8 pixels per cycle. The total number of CCD pixels required is the product of the DFT space bandwidth product with the number of pixels per frequency resolution element, which must be at least one cycle Thus $M=N \Delta f T / \gamma \Delta y>4 N \Delta f T$.

\section{TSI Folded Spectrum Based on TI DFT}

The characteristics of the TI DFT processor described in the previous section are complementary to those of the SI AO spectrum analyzer. The TI DFT processor is a narrow band processor with analysis bandwidth $\Delta f(1 / 2 T$, and high resolution of $1 / N T$ The SI AO spectrum analyzer is a broadband processor with bandwidth $B$, and resolution $\sim 2 / T_{a}$, which may also be limited by the CCD pixel size which has a frequency width of $v \Delta x / \lambda F$ Since the TI DFT processor is a 1-D system, we can spatially multiplex an array of such processors in the orthogonal dimension by using a 2-D CCD detector array Since each of the TI DFT processors in the array needs to perform the same narrow band spectrum analysis operation, and each signal is brought down to baseband by the pulsed laser diode, then they all can use the same reference AOD. One way that we can configure a 2-D acousto-optic folded spectrum processor is as an additive Mach-Zehnder interferometer with an SI AO spectrum analyzer to perform coarse frequency analysis in the $x$ dimension in one arm of the interferometer, and a spatially multiplexed array of TI DFT processors in the other arm in order to perform fine frequency analysis in the $y$ dimension on each coarse frequency bin. The fine frequency analysis bandwidth $\Delta f$ should be equal to the coarse frequency resolution per pixel in order to produce a full folded spectrum without any gaps.

The description and analysis of the TSI AO folded spectrum processor is made with reference to $F i g$, which schematically illustrates the Mach-Zehnder architecture in the upper half, and shows cross sections of the fine and coarse arms in the bottom half of the figure. The timing of the system is controlled by a $10 \mathrm{MHz}$ stable crystal oscillator that is digitally divided by 100 to produce a stable $100 \mathrm{kHz}$ pulse train used as the laser diode trigger. The laser diode is biased just below threshold and pulsed with a 50 nsec pulse every $10 \mu \mathrm{sec}$ The anamorphic Gaussian beam profile of the laser diode is collimated by the spherical lens and oriented with the long axis along the $x$ direction in order to optimally illuminate the entire SI AOD aperture. For the initial single tone experiments reported in this article the beam splitter 
reflects only about $10 \%$ of the light to the SI arm of the interferometer, because the processing gain associated with the spatial integration performed by the Fourler lens collects enough light to make this arm brighter at the output plane, so the optical efficiency can be increased by directing more light to the TI arm. For an actual SETI signal environment of broadband noise, the beam ratio can best be balanced through the use of a 50\% beam splitter. In the SI arm a cylındrical lens with power in $y$ focuses the light to a narrow slit positioned to coincide with the center of the diffracting acoustic beam, and incident on the AOD at the Bragg angle. The AODs used in this experiment are slow shear mode $\mathrm{TeO}_{2}$ devices with an aperture $T_{a}=70 \mu \mathrm{sec}$ and a bandwidth $B=40 \mathrm{MHz}$. The signal $s(t)$ applied to the transducer of AOD1 produces a diffracted component which is Fourier transformed in $x$ by the following spherical lens, which simultaneously recollimates in $y$. Thus each frequency component of $s(t)$ produces a uniform slit of light at the output plane, and the position of this slit varies linearly with the input coarse frequency. In the TI arm of the interferometer a cylindrical lens with power in $x$ is used to focus the light down to a narrow slit along $y$ which is incident on AOD2 at the Bragg angle. Only a $10 \mu \mathrm{sec}$ portion of the AOD needs to be uniformly illuminated with the narrow dimension of the LD elliptical beam profile. The reference signal is generated by incrementing a 10 bit digital counter with each LD pulse, and driving a 10 bit digital-to-analog converter in order to make a 1024 level staircase lastıng $10.24 \mathrm{msec}$. This signal is then applied to a phase resetable voltage controlled oscillator whose phase is reset each LD pulse producing the waveform $r(t)$ of Eq. (11), which is amplified and applied to AOD2. The diffracted signal from AOD2 is Fourier transformed by the spherical lens which recollimates in the $x$ dimension and allows a bandpass filter to be placed in the transform plane. The filtered light is retransformed in $y$ by the following cylinder, producing an mage of the signal in AOD2 with the approprate scale so that one $10 \mu \mathrm{sec}$ sinusold fills the entire CCD aperture $H$ in the $y$ dimension, and is uniformly spread out in the $x$ dimension. The final beam splitter recombines the two beams at an adjustable angle, and the $C C D$ time integrates the interferometric product between the two waves for a single video frame time of 167 msec. The CCD vertical synchronization signal is used to reset the 10 bit digital counter and begin another folded spectrum calculation by time integrating the next 1024 laser diode pulses.

The operation of the processor can be described analyt1cally by combining the results of the previous two sections The optical field incident on the CCD during the $n$th pulse is the sum of the reference wave acting as a linear spatial fre. quency modulation in the $y$ dimension, with the spatially Fourier transformed signal within the aperture of AOD1. The photogenerated charge profile detected by the CCD on each LD pulse is the modulus squared of the field incident during that pulse. Thus the time integrated interferometrically generated charge distribution recorded on the $\mathrm{CCD}$ after a full frame of processing is given by

$$
\begin{aligned}
I(u, y)= & \sum_{n=0}^{N-1} \mid b \mathrm{e}^{2 \pi(f+n \Delta f) y / v} \\
& +G \mathrm{e}^{j 2 \pi(\alpha+f / v) y} \times\left.\int_{A} f(x, n) \mathrm{e}^{-\jmath 2 \pi u x} d x\right|^{2} \\
= & N|b|^{2}+N\left|G \int_{A} f(x, n) \times \mathrm{e}^{-j 2 \pi u x} d x\right|^{2} \\
& +2 b G \sum_{n=0}^{N-1}\left[\int_{A} f(x, n) \mathrm{e}^{-\jmath 2 \pi u x} d x\right] \\
& \times \cos [2 \pi(n \Delta f y / v+\alpha y)]
\end{aligned}
$$

The phase reset reference wave starts at frequency $f$ and steps through $N$ discrete frequencies separated by $\Delta f$, and the angle of incidence in the $y$ direction $\Theta$ can be adjusted to make the resulting fringe structure at a desired spatial frequency $\gamma=\alpha+(N-1) \Delta f / 2 v$. The output pattern consists of a uniform bias term due to the reference beam, a signal dependent bias term with no varration in the $y$ dimension, and the last term which is recognized as the 2-D Fourner transform of $f(x, n)$, which is the desired folded spectrum of the long 1-D signal $s(t)$ The folded spectrum term is niding on a spatial carrier of spatial frequency $\gamma$ in the $y$ dimension so that it can be demodulated from the bias terms by an appropriate electronic bandpass filter. For the case of a single sinusoidal signal of frequency $f^{\prime}=k / T+\delta$, amplitude $|a|$, and phase $\Omega$, the output becomes

$$
\begin{aligned}
I(u, y)= & \sum_{n=0}^{N-1} \mid b \mathrm{e}^{j 2 \pi(f+n \Delta f) y / v} \\
& +G \mathrm{e}^{j 2 \pi(\alpha+f / v) y} \times\left. a Q(u-k / T v) \mathrm{e}^{j 2 \pi \delta n T}\right|^{2} \\
= & N|b|^{2}+N G^{2}|a|^{2} Q^{2}(u-k / T \nu) \\
& +2 G|a||b| Q(u-k / T \nu) \\
& \times N \operatorname{sinc}[N T(\delta-\Delta f y / H)] \\
& \times \cos (2 \pi \gamma y+\Omega)
\end{aligned}
$$


This pattern is the single tone impulse response of the system and is shown schematically in Fig. 6 It consists of a uniform bias term, a signal dependent bias ndge at the coarse frequency location $u=k / T \nu$, and the folded spectrum impulse of ampli. tude $|a|$ riding on top of the ridge at fine frequency position $y=\delta H / \Delta f$. The impulse is on a spatial carrier of frequency $\gamma$ in the $y$ dimension, with phase angle $\Omega$ which will vary on successive frames according to its super fine frequency $\Delta=$ res $_{2 / N T} \delta$; thus processing of successive frames can achieve even finer resolution. The resolution of Eq. (16) is given by the width of the sinc function and is limited by the integration time to $1 / N T$; however, the CCD spatial frequency response will apodize the DFT and decrease the achievable resolution. The fine frequency analysis bandwidth is seen to be $\Delta f$ as $y$ is allowed to vary over the CCD height $H$

Experimental results from this processor are shown in Fig. 7 for a fine frequency analysis bandwidth $\Delta f=4 \mathrm{kHz}$. The processor was operated with a coarse frequency resolution per pixel of $80 \mathrm{kHz}$, but due to limitations of the electronics, the fine frequency analysis bandwidth was limited to $10 \mathrm{kHz}$ and the data presented in Fig. 7 is therefore only a portion of a full folded spectrum. Cross sections along the bias ridge are shown in Fig. 7 (a-d) with different fine frequency inputs. The bras pedestal drops away near the edge of the output trace indicating the region of processor analysis $H=\nu T$. At a fine frequency difference of $120 \mathrm{~Hz}$ the constructive peak is quite large and well away from the edge. At a frequency of $240 \mathrm{~Hz}$ the destructive peak has moved over by about its width indicating that a resolution of $120 \mathrm{~Hz}$ or better has been achieved. At a frequency of $600 \mathrm{~Hz}$ the peak has moved over much farther but seems to be broadening. At $2100 \mathrm{~Hz}$ it is clear that the peak is broadening and decreasing in size. This appears to be due to the analog nature of the reference waveform generation introducing statistical frequency variations that produce larger phase uncertainties at higher frequencies This problem can be alleviated through the use of a digital buffer memory and digital to analog converter in order to produce the reference waveform However, this digital buffer would need to contain 1024 lines of 1024 words each that must be read out at a $100 \mathrm{MHz}$ rate, which is currently unavailable to us. For this reason an alternative architecture with a much simpler reference function was built for comparison purposes.

\section{Time Integrating Chirp Transform on TDI CCD}

A common method of performing spectral analysis with analog convolvers is the chirp transform algorithm. This algorithm results from the observation that the Fourier kernel can be decomposed into a convolution and two products through the use of the following identity

$$
-2 n m=(n-m)^{2}-n^{2}-m^{2}
$$

This expression can be substituted into Eq. (10), which defines the desired DFT, and rearranged to yield the chirp transform expression.

$$
\begin{aligned}
S(m)= & \sum_{n=0}^{N-1} s(n) \exp (-\jmath 2 \pi n m / N) \\
= & \exp \left(-j \pi m^{2} / N\right) \sum_{n=0}^{N-1}[s(n) \\
& \left.\times \exp \left(-j \pi n^{2} / N\right)\right] \exp \left(j \pi(n-m)^{2} / N\right)
\end{aligned}
$$

An examination of this representation indicates that we can accomplish a DFT by first pre-multiplying the input signal with a chirp, convolving the resulting product with a matched chirp, and finally post-multiplying the convolver output with a matched chirp in the Fourier domain. Often only the power spectrum is required in which case we can neglect the final post-multiplication by the quadratic phase factor, since its magnitude is unity everywhere.

There are a large number of methods for performing convolutions with time integrating optical techniques utilizing acousto-optics and charge coupled devices The features which must be incorporated in a convolver are an array of multipl-cations, a shift operation and an integration. An architecture we have used with great success for chirp correlations (Ref. 10) requires a temporally modulated input, a fixed chirp reference mask, and a CCD operated in the tıme delay and integrate (TDI) mode, where the detector time integrates in a shding coordinate frame. The CCD readout circuitry is modified so that it operates in a continuously scrolling fashion referred to as TDI or shift and add In this manner the CCD accumulates photogenerated charge at each pixel site for one or more laser pulses, the charge is then transferred vertically $(y)$ by one pixel of width $\Delta y$, and each pixel accumulates more charge, adding it to that previously detected. By the time a pixel has fully traversed the array of height $Y$, it has accumulated photogenerated charge from each position in space at successively delayed times If a temporally modulated sampled signal $f(n T)$ is uniformly incident on the chirp mask

$$
c(y)=\cos \left(b y^{2}\right) \operatorname{rect}(y / Y)
$$


and then imaged on the TDI CCD the resulting signal that scrolls off the edge of the detector is given by the convolution of the temporal signal with the spatially sampled image of the mask

$$
\begin{aligned}
I(m T) & =\sum_{n} f(n T) c(m \Delta y) * \delta(m-n) \\
& =\sum_{n} f(n T) c((m-n) \Delta y)
\end{aligned}
$$

The index $m$ enumerates the output samples obtained at the edge of the CCD at successive times $m T$. We can use this convolver to configure a tıme integratıng interferometric chirp transform power spectrum analyzer by introducing a quadratically phase modulated reference beam, and interfering this with a baseband phase modulated signal beam in order to perform an interferometric chirp pre-multiplication of the input signal as the detected intensity

$$
f(n T)=s(n T) \cos \left(b(n T)^{2} V^{2}\right)
$$

where $V=\Delta y / T$ is the TDI CCD shifting velocity The resulting time integrated power spectrum output would be obtained by demodulating the interferometric product term from the bias terms and squaring the chirp transform electronically. The analysis bandwidth of this type of TDI chirp transform spectrum analyzer is limited by the sampled mask resolution to half the CCD shift rate for each sideband of the reference chirp. A two sided chirp reference can cover an analysis bandwidth equal to the CCD shift rate $A$ feature of this type of chirp transform is that the spectral amplitude decreases linearly away from the center frequency as does the spectral resolution, due to a decrease in the matching chırp overlap.

The chirp transform spectrum analyzer can be readily incorporated in the TSI folded spectrum processor by including a quadratically phase modulated reference beam, and a chirp reference mask imaged onto a TDI CCD The resulting system architecture is shown schematically in Fig. 8 and can be seen to be just a modification of the previous optical system. The analytical description of the TSI folded spectrum system with the TDI chirp transform fine resolution processors results in the same signal and bias terms as in the previous system shown in Fig. 6. However the TDI approach has the advantageous feature that it averages coherent optical artifacts and CCD pixel noise along the shifting column thereby minımizıng the system fixed pattern noise. The system output is given by

$$
\begin{aligned}
F(u, m)= & \sum_{n=0}^{N-1} \mid \int_{A} f(x, n) \mathrm{e}^{-\prime 2 \pi u x} d x \\
& +\left.r \exp \left[-\jmath b(n T)^{2} V^{2}\right]\right|^{2} \\
& \times \cos \left(b(m \Delta y)^{2}\right) \operatorname{rect}[m \Delta y / Y] * \delta(m-n) \\
= & \sum_{n=0}^{N-1}\left[\int_{A} f(x, n) \mathrm{e}^{-\prime 2 \pi u x} d x\right] \\
& \times 2 r \cos \left[b(n \Delta y)^{2}\right] \cos \left(b((m-n) \Delta y)^{2}\right) \\
& \times \operatorname{rect}[((m-n) \Delta y) / Y]+\text { blas terms }
\end{aligned}
$$

We can see that the quadratically phase modulated reference beam results in an interferometric premultiplication of each sampled baseband coarse frequency bin with a temporal chirp. The combination of the chirp mask and the TDI CCD results in a chirp convolution for each coarse frequency channel, and the resulting folded spectrum raster scans out of the CCD as it scrolls. Experimental results from this processor are shown in Figs 9 and 10. Figure 9 (a-d) shows cross sections through the coarse frequency ridge of the impulse response of Fig. 6 for several input fine frequencies, and the decrease of peak height with increasing frequency is apparent. Figure 10 shows the 2-D Folded Spectrum output displayed on a video monitor for a variety of input frequencies, where the coarse ridge is seen to move with $10 \mathrm{MHz}$ frequency change, and $3 \mathrm{kHz}$ moves the fringes in the fine dimension along the ridge The spatial and temporal chirp references used in these experiments were two sided with 9 fringes per side giving a compression of 36 , and resulting in a spectrum analysis with 36 independently resolvable frequencies. The sideband structure is highly visible due to the hard clipping of the mask, both the spectral resolution and the linearity are better than $100 \mathrm{~Hz}$, and the performance closely matches the expected theoretical behavior.

\section{Demodulation of Signal From Bias Terms}

A crucial requirement for the successful operation of a TSI signal processing system is the ability to demodulate the signal from the bias terms. There are two main approaches to signal demodulation which are shown in Fig. 11, spatial carrier demodulation and CCD based demodulation. In carrier demodulation the interferometric signal term is placed on a spatial carrier by the adjustment of the reference beam incidence angle, while the bias terms remain at baseband When these signals are output from the CCD, a bandpass filter can sepa- 
rate the different components, and allow measurement of spectral amplitude and phase. The filtered output can be electronically squared and low passed in order to obtain a power spectrum, or rectification followed by a low pass can be used for measuring spectral amplitude components. The CCD raster readout mechanism can be used to change a spatial carrier in the horizontal direction into a fast temporal carrier that can be simply bandpassed by an active filter. A spatial carrier in the vertical dimension requires a more complicated set of video delay lines and a tapped filter in order to implement the desired bandpass operation. The spatial carrier can be placed in the coarse or fine frequency directions, but will cut down the avalable CCD space bandwidth product in whichever dimension it is used. In order to avold losing detector resolution, another possible approach is to have two synchronously operating CCD detectors, one to detect the interferometric signal and bias terms, and the other with just the signal term in order to detect the signal dependent bias. The two detectors must be precisely aligned and sub. tracted pixel by pixel in order to remove the signal dependent bias; the reference beam bias term should be uniform across the array and can be subtracted with a level shifter. An exper1mental bias subtraction technique we are investigating requires a special purpose CCD with a fill and spill structure abutted to the transfer register in order to bleed off an appropriate amount of charge to leave the signal term and a small amount of bias, rather than have the bias use up the available CCD dynamic range.

\section{AO Folded Spectrum Processor Performance}

A summary of the performance parameters for the TSI folded spectrum processor described in this article are given in Table 1, for the Caltech prototype, and for the potential of a fully engineered state of the art brassboard. The processor analysis bandwidth is determined by the AOD bandwidth, the CCD width and Fourier lens focal length, and by the laser diode pulse width at an acceptable level of illumination and temporal coherence. The Caltech prototype was limited by the CCD width, which can easily be modified The minimum laser diode pulse width is determined by the multımode transient operation for the first $1 \mathrm{nsec}$ which results in incoherent operation inappropriate for this processor (Ref. 11); however, light limitations will also dictate a minimum pulse width determined by peak output power. The number of coarse frequency bins is determined primarily by the number of CCD pixels, but another limitation is due to the AOD time bandwidth product and apodization unformity. The avalability of the Tektronix $2000 \times 2000 \mathrm{CCD}$ is very encouraging for the development of optical processors requiring high resolution 2-D detectors. The coarse frequency resolution is limited by the AOD transit time and apodization, but also determined by the FT lens focal length and pixel width. The number of fine frequency resolution bins is determined by the space bandwidth of the reference functions and the num. ber of CCD pixels available. If a spatial carrier is included in the fine frequency dimension, then the number of fine resolution bins is further limited, and a maximum spatial carrier frequency of 4-6 pixels per cycle indicates that a maximum of 500 fine frequency bins could be predicted The fine resolution is fundamentally limited by the CCD integration time during which the LD is being pulsed, and the prototype processor has closely approached this limit. Actually a greater difficulty is encountered in attempting to acheve a fine frequency analysis bandwidth equal to the coarse frequency resolution, which requires high bandwidth electronic reference generation currently not obtainable with our prototype circuitry. The TDI chirp transform approach is limited in analysis bandwidth by the mask resolution and by the obtainable CCD shift rate, which is currently limited to $15 \mathrm{kHz}$ for video compatible devices, but could be increased with special CCD circuitry. The total number of independent spectral measurements made in our intial experiments was only 15,000 but a potential for on the order of $10^{6}$ clearly exists. The dynamic range is currently quite small due to coherence limitations and light starvation, but this will be greatly improved in the next generation processor. The most important performance parameter for the SETI search is the sensitivity to a signal buried in noise, and the processing gain limitations of the analog technique used to perform the spectral analysis The large AOD dynamic range allows very weak signals to be effectively transduced into the optical processor, but a huge CCD dynamic range is not required to achieve the desired processing gain, since the spatial integration achieves much of it.

Since sensitivity and processing gain are so important to the SETI application we will include a simple analysis of the processor limitations based on the model presented in Fig. 12. For a weak narrowband $C W$ input burled in white noise with power $\sigma_{n}^{2}$ we will have a system input

$$
s(t)=a \mathrm{e}^{j \omega t}+n(t)
$$

The amplifier and AOD have a large linear region and a saturation regime that will introduce third order intermodulation products that will degrade system performance An ideal gain $G$ is included due to an optical power increase which will not increase the relative power in the intermodulation terms. The processor consists of an SI coarse frequency channelizer which acts as $10^{3}$ bandpass filters (BPF), cascaded onto an array of $10^{3}$ TI spectrum analyzers, resulting in the synthesis of $10^{6}$ narrow band filters. The detected spectral amplitudes are corrupted by the inclusion of signal dependent nosse, and the detector noise term with variance $\sigma_{d}^{2}$. The processing gain 
1s defined as the ratio of input signal to noise ratio (SNR) to output SNR, and ideally it would be given by

$$
P G=\frac{S N R_{\text {out }}}{S N R_{\text {in }}}=\frac{a^{2} / \beta^{2} \sigma_{n}^{2}}{a^{2} / \sigma_{n}^{2}}=1 / \beta^{2} \sim 10^{6}
$$

where $\beta^{2}$ is the BPF width divided by the entire bandwidth, which is equivalently the inverse of the number of channels After the demodulation operation, which will remove the bias terms, including the bias shot noise contribution in $\sigma_{d}^{2}$, the expected value of the power spectrum component at the frequency of the input tone will be

$$
\left\langle S_{\omega}^{2}\right\rangle=a^{2} G^{2}+\beta^{2} G^{2} \sigma_{n}^{2}+\sigma_{d}^{2}
$$

The input sensitivity is determined by the minımum detectable signal at the desired false alarm probability, which for SETI is on the order of $10^{-10} \sim \mathrm{e}^{-20}$, in order to keep the total number of false alarms manageable By cross scanning the sky and comparing positions and frequencies of spectral components exceeding the given threshold, the false alarm probability can easily be squared. Thus the minımum detectable signal resulting in a false alarm probability of $e^{-10}$ would be 10 standard deviations above the noise

$$
a_{\min }^{2} G^{2}>10\left[\beta^{2} G^{2} \sigma_{n}^{2}+\sigma_{d}^{2}\right]
$$

To minimize effect of the detector noise limitations we increase the gain $G$ to the point that the signal dependent noise and the detector noise are comparable. In this case minimum input SNR that can result in a signal exceeding the threshold is given by

$$
\begin{aligned}
\mathrm{SNR}_{\min } & =a_{\min }^{2} / \sigma_{n}^{2} \\
& =10\left[\beta^{2}+\sigma_{d}^{2} / G^{2} \sigma_{n}^{2}\right] \sim 20 \beta^{2}
\end{aligned}
$$

Thus the resulting input sensitivity is degraded by $13 \mathrm{~dB}$ from the initial $-60 \mathrm{~dB}$, but only $3 \mathrm{~dB}$ of the degradation are an artifact of the analog optical processor. This estımate for the actual input sensitivity of $-47 \mathrm{~dB}$ will be further degraded by the intermodulation product terms due to the amplifier and AOD nonlinearities, by the shot noise due to the bias, and probably most importantly by light starvation limiting the gain $G$

\section{Conclusion}

In this article we have presented prelıminary experımental results from time and space integrating folded spectrum processors that may have potential application in the SETI program. The sampling action of the repetitively pulsed laser diode eliminates the need for a distributed local oscillator reference wave by aliasing all of the fine frequency components to baseband at the output of the coarse frequency channelizing space integrating spectrum analyzer as is required for the following CCD based narrow band time integratıng spectrum analyzers. Two methods of time integrating fine frequency analysis were presented, the time integrating DFT, and the time delay and integrate chirp transform. The TI DFT has the attractive possibility for incoherent addition of spectra in order to improve SNR, but requires a very complex and accurate reference waveform. The TDI chirp transform helps average out coherent noise and detector noise, and has a simple reference function, but does not allow simple complex spectral demodulation, and is bandwidth limited by the avallable CCD shift rate The possible advantage of this technıque over alternative digital approaches is a decrease in system cost and complexity, along with the elimination of the high speed analog to digital converter required for digital processing. For a possible future spaceborne SETI search the optical processor has decreased size, weight and power requirements over that of a digital approach 


\section{Acknowledgments}

This work was performed under grants from the AFOSR and NASA and an ARO graduate research fellowship at Caltech. We thank Sam Gulkis and the entıre SETI science working group for introducing us to SETI and partially motivating this work We also thank Mike Haney for his collaboration on TSI optical processing.

\section{References}

1. Drake. F., Wolfe, J.H, and Seeger, C. L, Eds., "SETI Science Workıng Group Report," NASA Tech Paper 2244, Washington, D.C., Oct. 1983.

2. Thomas, C. E., "Optical Spectrum Analysis of Large Space Bandwidth Signals," Appled Optics, vol. 5, pp. 1782-1790, 1966.

3. Turpin, T. M., "Spectrum Analysis Using Optical Processing," Proc. IEEE, vol. 69, pp 79-92, Jan. 1981.

4. Kellman, P, "Time Integrating Optical Signal Processing," Ph D. dissertation, Stanford Univ., Stanford Ca., 1979.

5. Bader, T. R., "Coherent Optical Hybrid Techniques For Spectrum Analysis," Proc. $S P I E$, vol. 185, pp. 140-146, 1979.

6 Bader, T. R., "Acousto-Optic Spectrum Analysis: A High Performance Hybrid Technique," Applied Optics, vol. 18, pp. 1668-1672, 1979

7. Psaltis, D., and Casasent, D., "Time and Space Integrating Spectrum Analyzer," Applied Optics, vol. 18, pp. 3203-3204, 1979.

8. Psaltis, D., "Two Dimensional Optical Processing Using One-Dimensional Input Devices," Proc. IEEE, vol 72, pp. 962-974, 1984

9. Van der Lugt, A., "Interferometric Spectrum Analyzer," Applied Optics, vol. 20, pp 2770-2779, 1981.

10. Psaltis, D., and Wagner, K., "Real-Time Optical SAR Processor," Optical Eng., vol. 21, pp. 822-828, 1982.

11. Haney, M., and Psaltis, D., "Measurement of the Temporal Coherence Properties of Pulsed Laser Diodes," Applied Optics, vol. 24, pp 1926-1932, 1985. 
Table 1. AO processor performance

\begin{tabular}{|c|c|c|}
\hline Parameter & $\begin{array}{l}\text { Caltech } \\
\text { Prototype }\end{array}$ & Potential \\
\hline$B=$ Bandwidth & $30 \mathrm{MHz}$ & $1 \mathrm{GHz}$ \\
\hline$n_{c}=$ Course Bins & 384 & 2000 \\
\hline$f_{c}=$ Course Resolution & $80 \mathrm{kHz}$ & $B / 1000$ \\
\hline$n_{f}=$ Fine Bins & $\begin{array}{l}36 \text { (actual) } \\
491 / a_{\text {carrier }} \\
\text { (potential) }\end{array}$ & $2000 / a_{\text {carrier }}$ \\
\hline$f_{f}=$ Fine Resolution & $\begin{array}{l}120 \mathrm{~Hz} \text { (actual) } \\
1 \mathrm{kHz} \text { (potentral) }\end{array}$ & $f_{c} / 1000$ \\
\hline$N=$ Spectral Resolution & 15,000 & $10^{6}$ \\
\hline$D R=$ Dynamic Range & 50 levels & 1000 levels \\
\hline Sensitivity & $-40 \mathrm{~dB}$ & $-60 \mathrm{~dB}$ \\
\hline
\end{tabular}


(a)

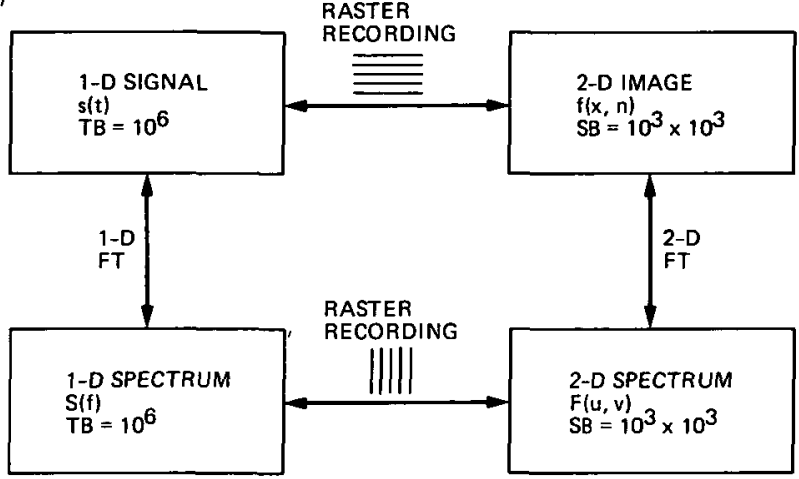

(b)

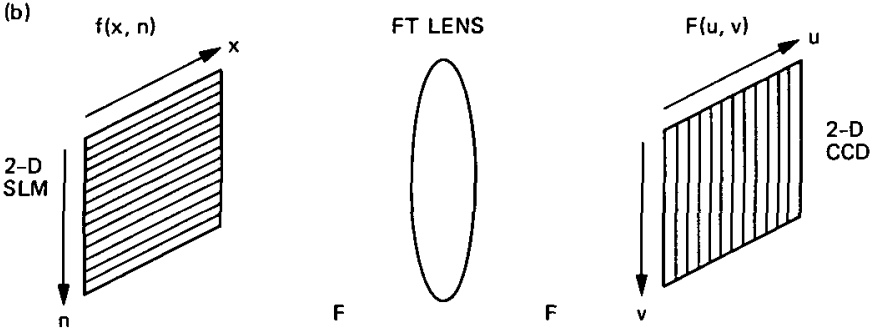

Fig. 1. Two-dimensional space integrating folded spectrum processing: (a) the relationship between a long 1-D signal, its high resolution spectrum, the folded spectrum, and the signal's raster representation; (b) a space integrating folded spectrum processor.

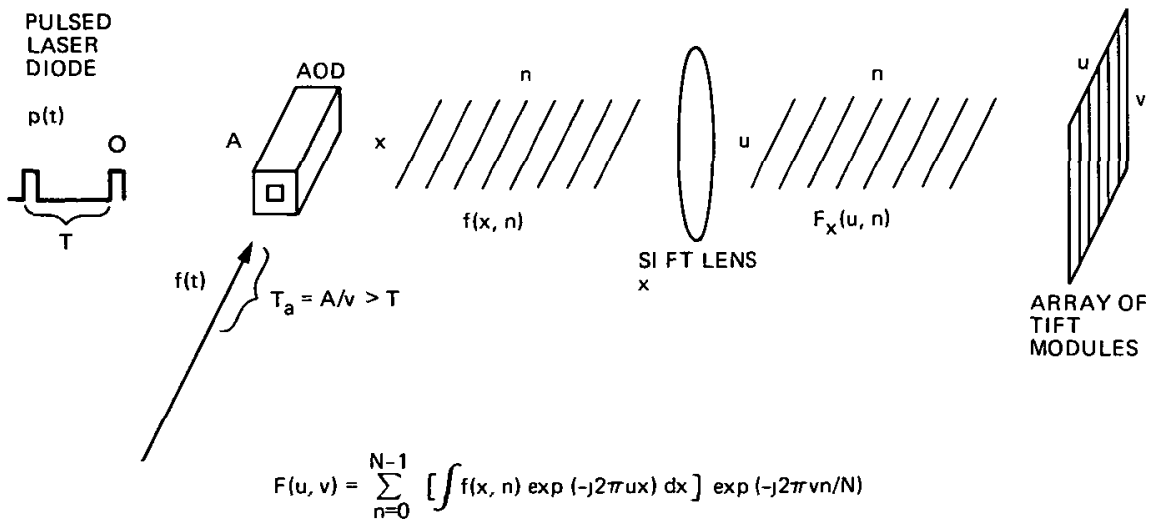

Fig. 2. Time and space integrating folded spectrum processor showing the conversion to a space-time raster, spatial Fourier transformation cascaded onto an array of temporal Fourier transforms. 


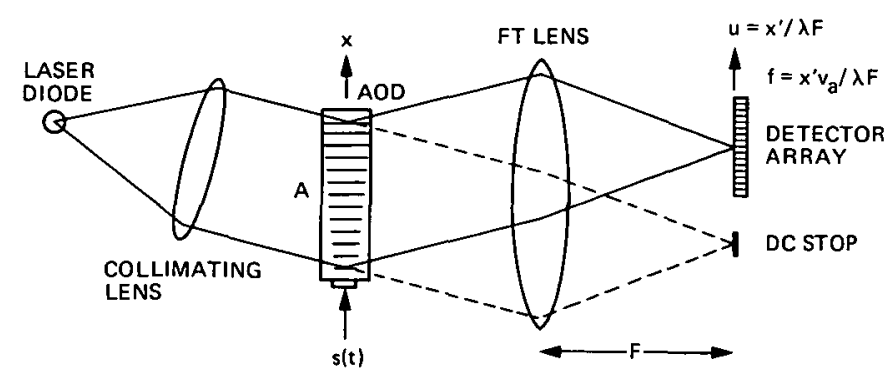

Fig. 3. The space integrating acousto-optic spectrum analyzer

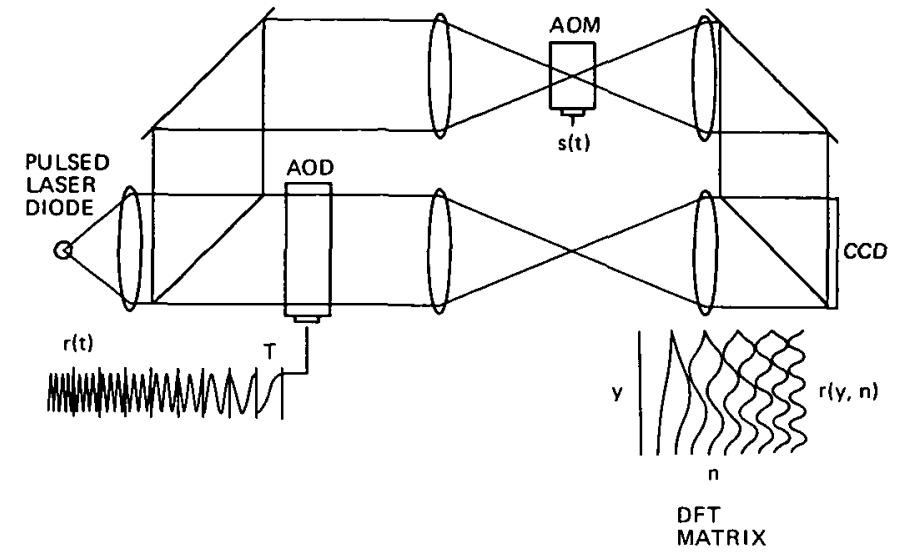

Fig. 4. Interferometric time integrating DFT narrow band spectrum analyzer with phase locked stepped frequency reference

(a)
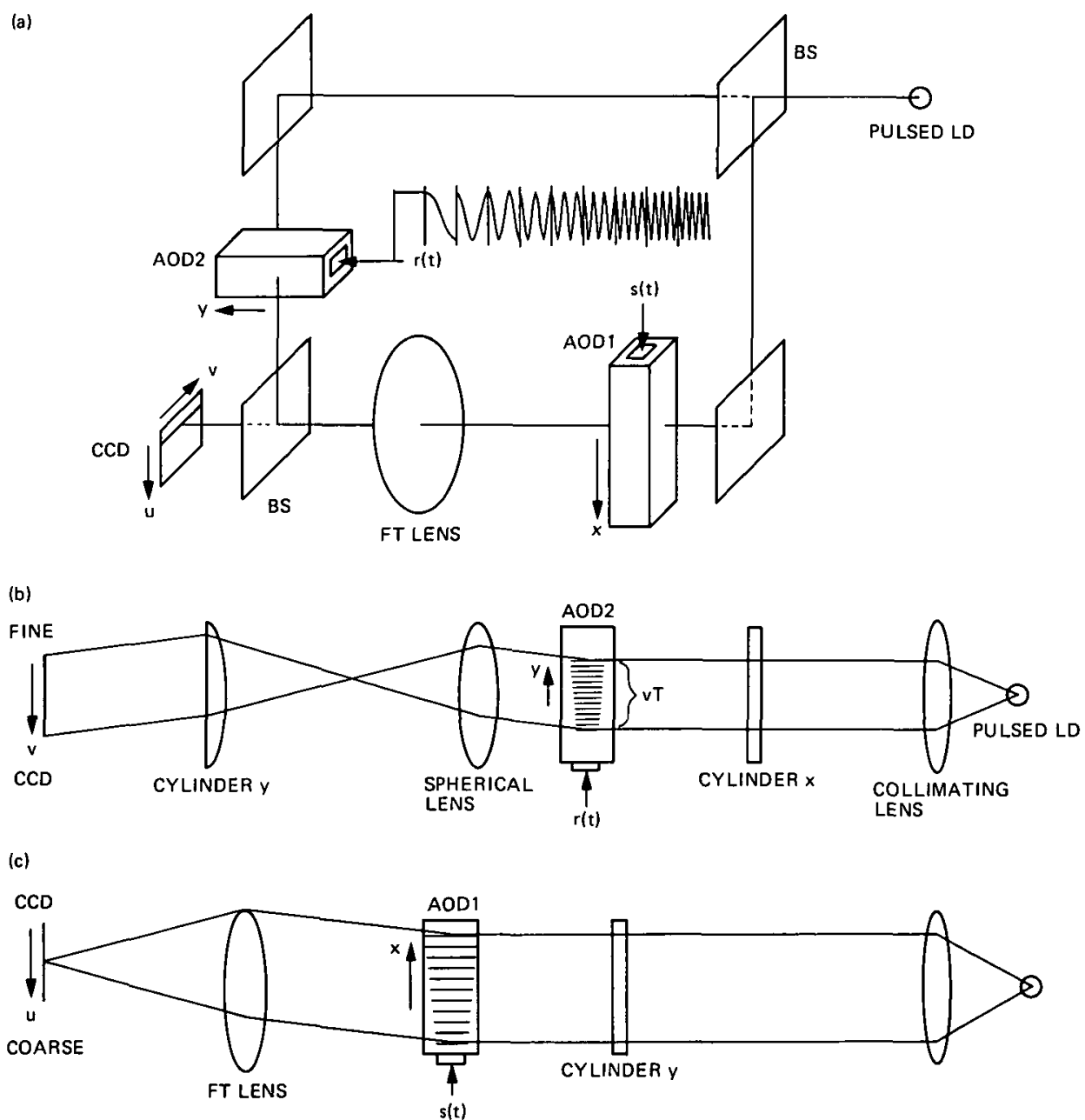

Fig. 5. The Mach-Zehnder interferometer architecture: (a) for performing the TSI folded spectrum; (b) cross section through $\mathrm{Tl}$ fine resolution spectrum analyzer arm; (c) cross section through SI coarse resolution spectrum analyzer arm 
ORIGINAL PAGE IS

\section{OF POOR QUALTTY}

COARE FREQUENCY AXIS

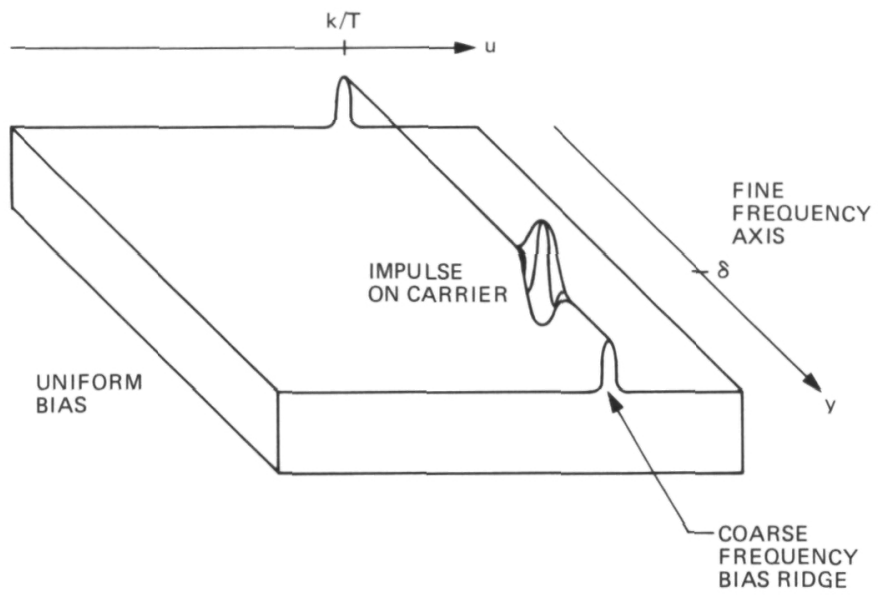

Fig. 6. Impulse response for TSI folded spectrum processor for a single tone input at frequency $\boldsymbol{f}^{\prime}=\boldsymbol{k} / \boldsymbol{T}+\delta$, showing uniform bias, signal dependent bias, and impulse riding on carrier

Fig. 7. Experimental results for the TSI folded spectrum processor of Fig. 5, showing cross sections through the coarse frequency ridge for various fine frequencies (a) $\delta f=120 \mathrm{~Hz}$

(b) $\delta f=240 \mathrm{~Hz}$

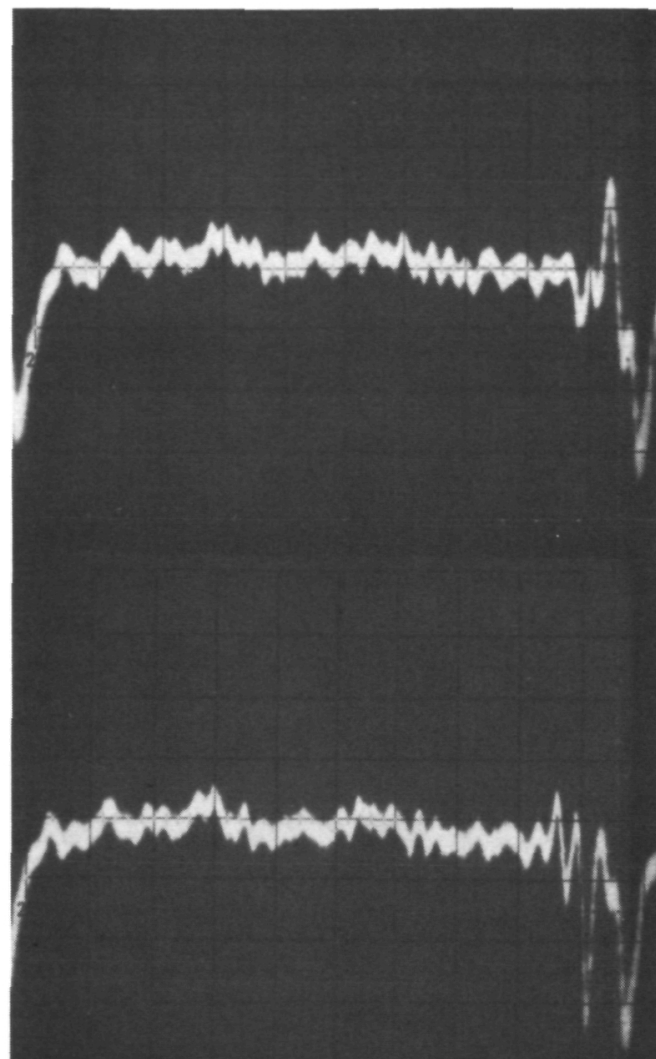

(c) $\delta f=600 \mathrm{~Hz}$

(d) $\delta f=2100 \mathrm{~Hz}$
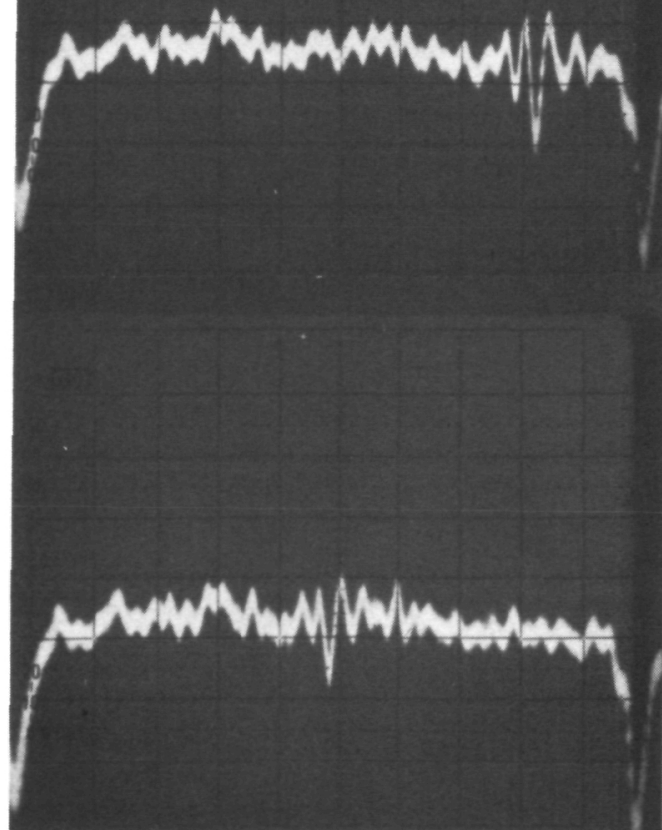
$F(u, v)=\sum_{n=0}^{N-1}\left|\int f(x, n) \exp (-12 \pi u x) d x+r \exp \left[-J b(n T)^{2} v^{2}\right]\right|^{2} \cos \left(b y^{2}\right) * \delta(n T-v / V)$

SI REFERENCE CHIRP $\underset{\text { MASK }}{\text { CHIRP }}$ CDI

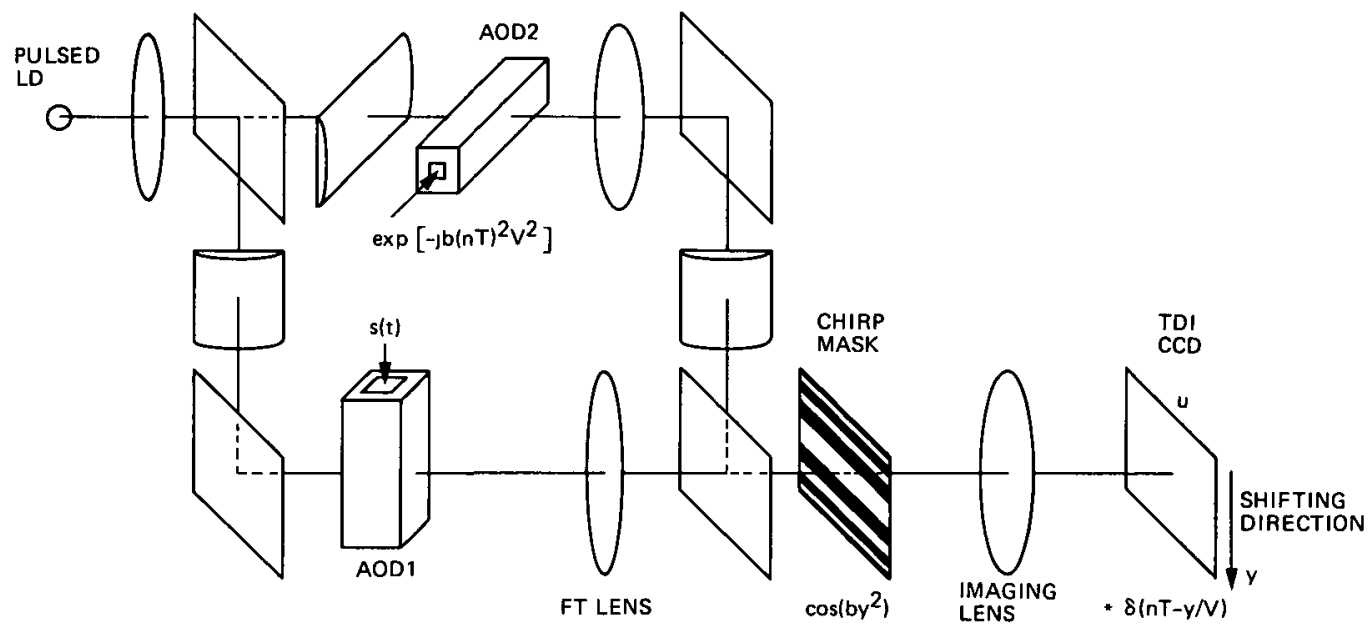

Fig. 8. Architecture for performing TSI folded spectrum using a TDI CCD to perform a fine resolution chirp transform 


\section{ORIGINAL PAGE IS \\ OF POOR QUALITY}

(a) $\delta f=-100 \mathrm{~Hz}$

(b) $\delta f=-1000 \mathrm{~Hz}$

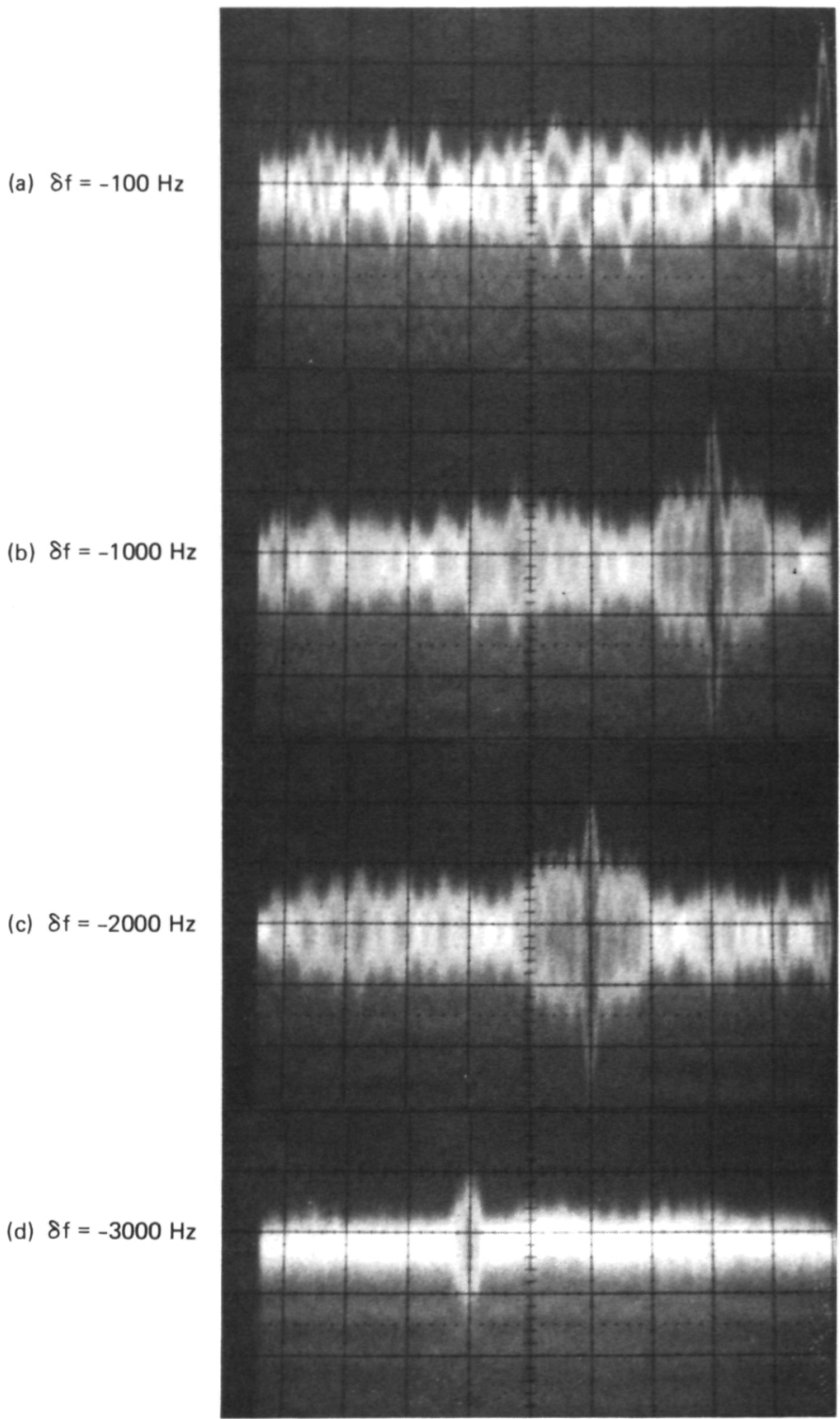

(d) $\delta f=-3000 \mathrm{~Hz}$

(c) $\delta f=-2000 \mathrm{~Hz}$

Fig. 9. Cross sections through coarse frequency ridge for the folded spectrum processor of Fig. 8 for various fine frequencies 


\section{ORIGINAL PAGE SS \\ OF POOR C':AOTY}

\section{ORIGINAL PAGE IS \\ OF POOR QUALITY}
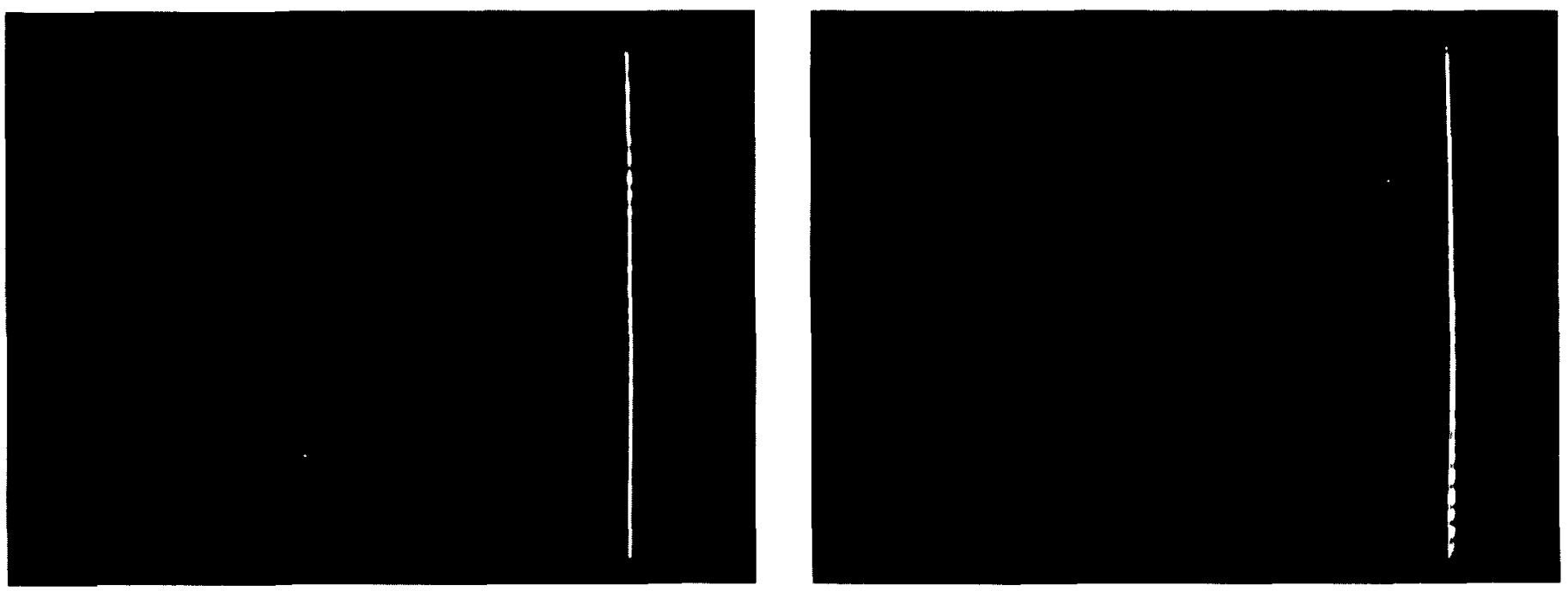

(a) $60000 \mathrm{MHz}$

(b) $60003 \mathrm{MHz}$

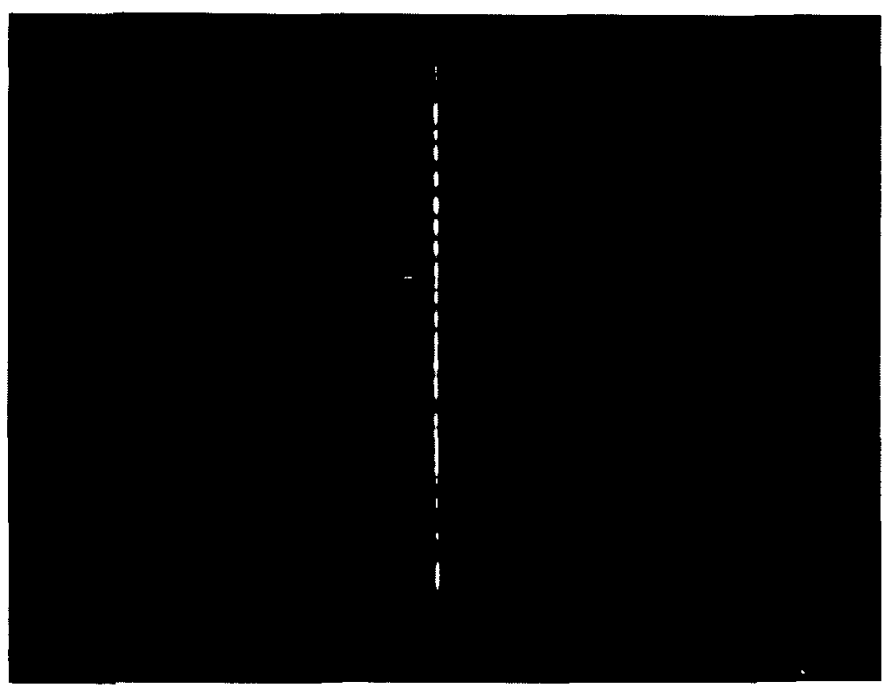

(c) $50000 \mathrm{MHz}$

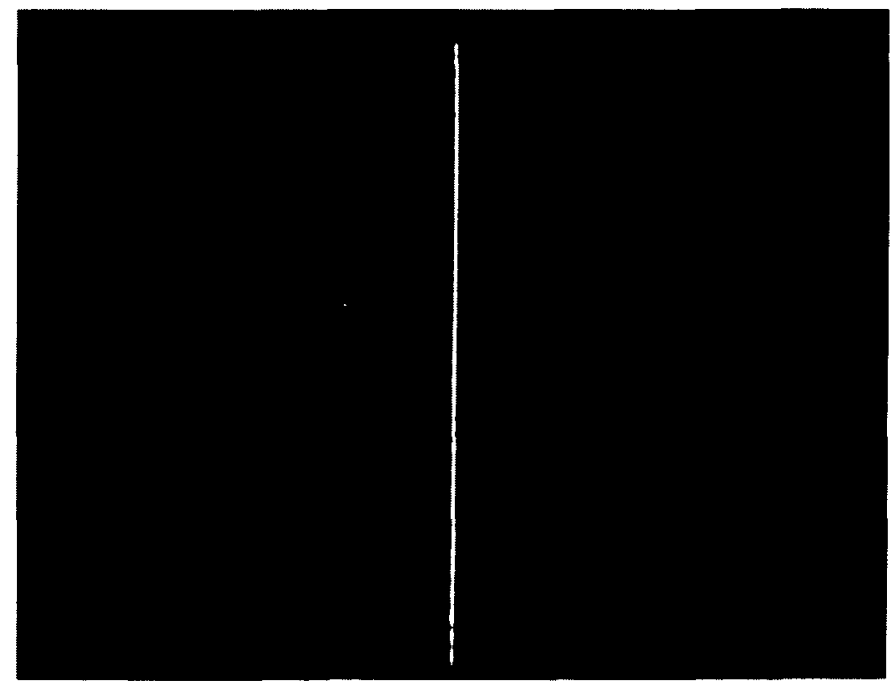

(d) $50003 \mathrm{MHz}$

Fig. 10. Impulse response of TSI folded spectrum processor displayed in a two-dimensional format on a video monitor 


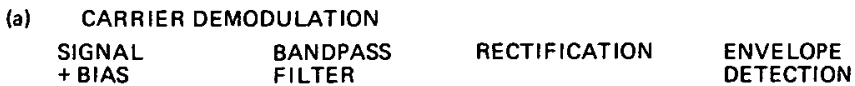

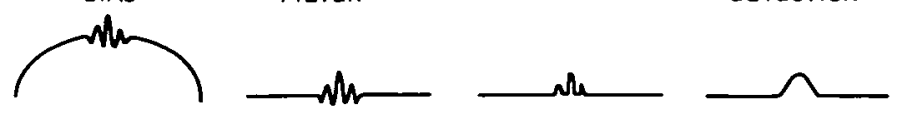

(b) HORIZONTAL

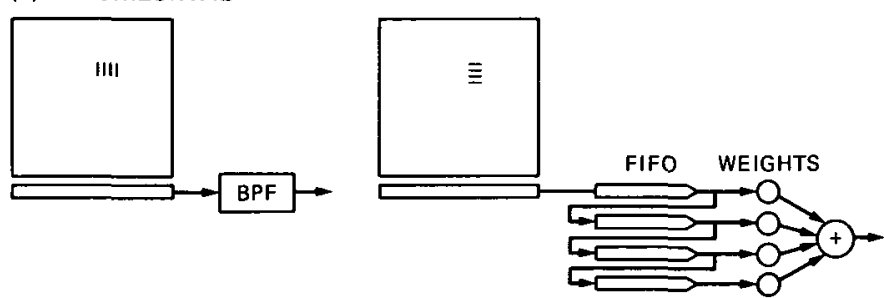

(c) BIAS SUBTRACTION WITH 2 CCDs

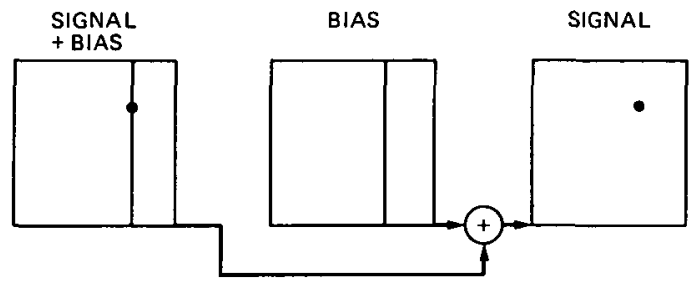

(d) FILL AND SPILL ADAPTIVE BIAS SUBTRACTION CCD

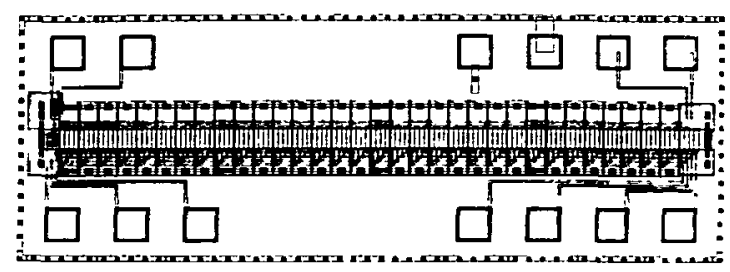

Fig. 11. Demodulation schemes required to separate signal from bias terms

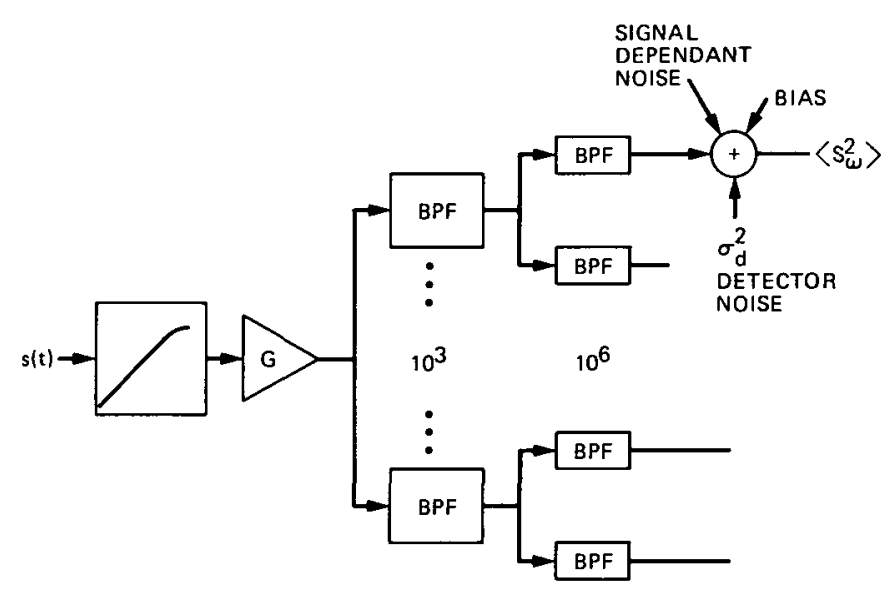

Fig. 12. Model used for sensitivity and processing gain analysis 